Commendatore P, Kubin I, Mossay P. On the new economic geography of a multicone world. Review of International Economics 2017, epub ahead of print.

DOI link

https://doi.org/10.1111/roie.12312

ePrints link

http://eprint.ncl.ac.uk/pub details2.aspx?pub id=241188

Date deposited

$08 / 12 / 2017$

Embargo release date

$26 / 09 / 2019$

Copyright

This is the peer reviewed version of the following article: Commendatore P, Kubin I, Mossay P. On the new economic geography of a multicone world. Review of International Economics 2017, which has been published in final form at https://doi.org/10.1111/roie.12312. This article may be used for non-commercial purposes in accordance with Wiley Terms and Conditions for Self-Archiving. 


\title{
On the new economic geography of a multi-cone world
}

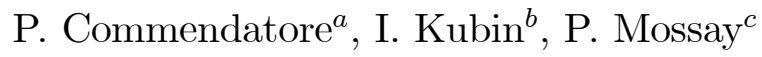 \\ ${ }^{a}$ University of Naples 'Federico II', Italy \\ ${ }^{b}$ Vienna University of Economics and Business Administration, Austria \\ ${ }^{c}$ Newcastle University, UK, and CORE, Belgium
}

March 12, 2017

\begin{abstract}
We build a New Economic Geography model incorporating differences in productivity among sectors and countries, thus allowing for comparative advantage. We study the role that market size, absolute advantage, and comparative advantage have on the trade patterns and the long-run spatial distribution of economic activity in a world with multi-cones of specialisation. We briefly mention the possibility of long-term fluctuations in the spatial distribution of industry by relying on a discrete-time framework.
\end{abstract}

\section{Introduction}

In an influential work, Krugman (2008) highlights that as developing countries engage further in trade in industrial commodities, they tend to specialise in manufactured commodities differing from those industrialised countries specialise in. This notion of mutiple cones of specialisation, dating back to Leamer (1987), has gained increasing attention in the analys of international trade patterns (for recent evidence see e.g. Kiyota, 2014). At the same time, firms' market power and increasing returns to scale are known to affect industrial production and its location, especially in a globalised world where productive factors become increasingly mobile. The main motivation of our paper is to reconcile the imperfect competition modelling of core-periphery structures with the evidence on multi cones of specialisation.

New Economic Geography (NEG) models rely on the presence of increasing returns to scale in production and factor mobility to explain the spatial agglomeration of economic activity and the emergence of core regions and peripheries, see e.g. Krugman (1991), Fujita et al. (1999). When agglomeration takes place, larger markets tend to attract further economic activity. On the other hand, classical models of trade based on Comparative Advantage (CA) rely on 
differences in productivity among regions or countries to explain specialization patterns.

The aim of the paper is to analyze the role that Absolute Advantage (AA) and CA have on the spatial distribution of economic activity in the presence of market size effects in a multi-cone world. For that purpose, we develop a new economic geography model where differences of productivity across countries, providing them with $\mathrm{AA}$ and $\mathrm{CA}$, interact with the agglomeration force driven by increasing returns to scale.

Some contributions in the literature deal with CA and industrial concentration within a New Economic Geography framework. In his seminal work Ricci (1999) introduces two manufacturing sectors in Krugman (1991)'s CorePeriphery (CP) model. A country can have a CA or an AA in the production of one of the manufactured goods, as reflected by a lower marginal cost of production (either in relative or absolute terms). In Ricci's (1999) analysis, CA has a positive direct effect on specialization - firms of a given sector are attracted by the country which has a CA in that sector -; as well as a negative indirect effect: an increase in market size induced by the influx of firms of the sector where the country has a CA implies lower specialization as firms of the other sector move to that country as well. So a 'perverse' relationship between CA and specialization may occur (but not a complete reversal). Moreover, CA could act as dispersion force: so that a reduction in trade costs does not necessarily lead to more agglomeration, depending on the counterbalancing of CA (and AA) and market size. Moreover, the possibility of stable multiple equilibria could lead to partial agglomeration in the less productive country (in AA terms). In Ricci (1999), full specialization is not considered as both countries are assumed to be producing both goods. Moreover, congestion externalities are introduced so as to avoid full agglomeration of the industrial activity in either country. ${ }^{1}$ Ricci's (1999) analysis is further developed by Forslid and Wooton (2003), in a slightly different $\mathrm{CP}$ model where firms in the same sector are characterised by different fixed production costs. ${ }^{2}$ Differences in productivity also depend on where firms locate, thus allowing for $\mathrm{CA}$ in each county over a range of product varieties. Forslid and Wooton (2003) show that the counterbalancing of CA and agglomeration forces, especially driven by market size, is affected by trade integration

\footnotetext{
${ }^{1}$ Bagoulla (2006) reformulates Ricci's analysis in the framework of the Footloose Capital (FC) model of Martin and Rogers (1995). Her model considers a North-South economy characterised by CAs in the sector with high added-value (North) and with low added-value (South), wage differentials and differences in the quality of infrastructures. Regarding the 'perverse' relationship between specialization and CA, Bagoulla confirms Ricci's (1999) result. Moreover, this author also excludes by assumption the possibility of full specialization. Finally, full agglomeration cannot occur either. Matsuoka and Kikuchi (2012) also put forward a FC model aiming to study the combined effect of differences in market size and technological advantages. They found that when one of the countries is sufficiently large, agglomeration can occur in the country which has a comparative disadvantage but enjoys a larger local demand. In their model however, there is only one increasing returns sector and the technological difference only exists between the two countries.

${ }^{2}$ Huang et al. (2014) assume that both fixed and marginal costs may differ across countries, but not across varieties. These authors show that the home market effect could be overcome by $\mathrm{CA}$ when the small country has a technological edge.
} 
in the following way: at high trade costs, the economic activity is fully dispersed due to the prevalence of local demand; at low trade costs, where agglomeration forces are weak, CA representing a dispersion force (firms are attracted symmetrically where they can be more productive) overcomes the market size effect, so that again, industrial activity is fully dispersed. For intermediate trade costs, agglomeration forces are sufficiently strong to prevail over the dispersion effect induced by CA, so that partial agglomeration occurs. Note that the existence of multiple stable equilibria is obtained, with smooth transition in two directions: towards higher agglomeration or towards full dispersion. Finally, both full specialization and full agglomeration are excluded, since the incentive for a firm to locate in the country where it is more productive increases rapidly as the number of firms in that country decreases. Finally, in a quite different set-up, Pflüger and Tabuchi (2016) are able to disentangle the effects of CA and market size. They consider a vertically integrated economy, where CA and constant returns to scale characterise the final good sector and increasing returns to scale the sector producing intermediates. For our purposes, their most interesting result is the combined effect from reducing simultaneously the costs of trading final and intermediate varieties, which are assumed to be different. In Pflüger and Tabuchi (2016), agglomeration forces are enhanced by reducing the costs of trading the intermediates, whereas lowering trade costs in the final good sector enhances the effect of CA, which is a dispersion force favouring the spreading of economic activity. The simultaneous increase of trade freeness in these two sectors leads to their spatial concentration. Full agglomeration can only occur in the intermediate good sector, with both the Core and the Periphery producing the final good, although in different proportions.

Our framework extends the two-country Foootlose Capital model by Martin and Rogers (1995) to the case of two monopolistically competitive sectors. This provides a more tractable model than that by Ricci (1999) who used a similar extension in the context of Krugman's CP model. Unlike traditional NEG models, the Footlose Capital model does not exhibit circular causality as location of demand is not endogenous, see Baldwin et al. (2005) and Head and Mayer (2004). As in Ricci, the labor requirements vary across sectors and countries so that each country has a CA in a particular sector. In the short-run equilibrium, the international distribution of capital determines the specialization patterns across countries. So as to focus on multi cones of specialisation, we depart from Ricci by studying trade patterns where at least a country fully specialises in a single manufacturing sector. Based on capital remunerations available across countries, capital tends to relocate to the most profitable country. We look at how the dynamics and the resulting long-run equilibria are affected by CA, AA and market size effects.

We obtain the following results: in the short run, CA determine the specialization of trade patterns. In the long run, AA determines the relocation of production factors, and thus the location of agglomeration. This result differs from standard symmetric CP models where agglomeration can occur in any country. In addition, when varying trade freeness, transition to full agglomeration is smooth, which contrasts with the abrupt transition provided by many 
NEG models. We conclude with a short section on more complex dynamic phenomena: we find coexistence of stationary stable equilibria, in particular of an interior equilibrium (with some dispersion of the manufacturing activity across countries) and a $\mathrm{CP}$ equilibrium; and, in a discrete time set-up, the presence of long-term regular or complex cyclical adjustments in the distribution of the economic activity across space.

We structure the paper as follows. Section 2 presents the Footlose Capital model with two manufacturing sectors. In Section 3 we study how a distribution of capital across countries determines the trade specialization patterns in a multi-cone world. Section 4 studies the long-run dynamics induced by differences in capital remunerations across countries. In particular, we stress the role that $\mathrm{CA}, \mathrm{AA}$, and market size have on the location of economic activity. Section 5 concludes.

\section{Model}

The model extends the Foootlose Capital model by Martin and Rogers (1995) to the case of two monopolistically competitive manufacturing sectors $X$ and $Y$. The economy consists of 2 countries, $i=1,2$. Two factors of production are used: labor and capital. Labor is evenly spread across countries $L_{1}=L_{2}=L / 2$. The share of total capital $K$ used in country $i$ is denoted by $k_{i}$. Capital owners collect their remuneration on capital in the country where it is used and spend it in their country of origin. As capital income is assumed to be the same in both countries, world income is also equally distributed across space: $I_{i}=I_{W} / 2$ still to be determined.

Individuals have the following upper tier utility function determining the choice between the agricultural good and the varieties produced in the two manufacturing sectors:

$$
\begin{aligned}
U & =X^{\gamma \mu} Y^{(1-\gamma) \mu} A^{1-\mu} \\
0 & <\gamma, \mu<1
\end{aligned}
$$

where $\mu$ is the share of manufacturing expenditure, $A$ the consumption of the agricutural good, $\gamma($ resp. $1-\gamma)$ indicating the share spent on the manufacturing aggregate $X($ resp. $Y)$ and a lower tier determining the choice across the varieties produced in the two manufacturing sectors:

$$
X=\left(\sum_{s=1}^{N_{X}} x_{s}^{\frac{\sigma-1}{\sigma}}\right)^{\frac{\sigma}{\sigma-1}}, Y=\left(\sum_{s=1}^{N_{Y}} y_{s}^{\frac{\sigma-1}{\sigma}}\right)^{\frac{\sigma}{\sigma-1}}
$$

where $\sigma$ is the elasticity of substitution among varieties of the same manufacturing sector; $N_{X}\left(\right.$ resp. $\left.N_{Y}\right)$ the number of varieties produced in sector $X$ (resp. $Y)$.

Following Ricci (1999), we introduce Ricardian competitive advantages by differentiating variable costs across sectors and countries. With techological 
requirements for a firm in sector $X$ (resp. $Y$ ) in country $i$ of 1 unit of capital and $\beta_{X_{i}}$ (resp. $\left.\beta_{Y_{i}}\right)$ workers to produce $q_{X_{i}}\left(\right.$ resp. $\left.q_{Y_{i}}\right)$ units of a variety of $X$ (resp. $Y$ ), the cost functions can be written as

$$
C_{X_{i}}\left(q_{X_{i}}\right)=\pi_{X_{i}}+\beta_{X_{i}} q_{X_{i}} \quad ; \quad C_{Y_{i}}\left(q_{Y_{i}}\right)=\pi_{Y_{i}}+\beta_{Y_{i}} q_{Y_{i}}
$$

where $\pi_{X_{i}}$ (resp. $\pi_{Y_{i}}$ ) denotes the remuneration of capital in sector $X$ (resp. $Y)$.

Monopolistic pricing in the two sectors in the two countries leads to

$$
p_{X_{i}}=\beta_{X_{i}} \frac{\sigma}{\sigma-1} ; p_{Y_{i}}=\beta_{Y_{i}} \frac{\sigma}{\sigma-1}
$$

Price indices in manufacturing sectors can then be written as

$$
P_{X_{i}}^{1-\sigma}=p_{X_{i}}^{1-\sigma} K\left(h_{X_{i}} k_{i}+\phi h_{X_{j}}\left(1-k_{i}\right)\right) ; P_{Y_{i}}^{1-\sigma}=p_{Y_{i}}^{1-\sigma} K\left(h_{Y_{i}} k_{i}+\phi h_{Y_{j}}\left(1-k_{i}\right)\right)
$$

where $0 \leq h_{X_{i}}, h_{Y_{i}} \leq 1$ are the shares of capital used in sectors $X$ and $Y$ in country $i$ such that $h_{X_{i}}+h_{Y_{i}}=1$.

Free entry of firms fixes the capital remunerations

$$
\pi_{X_{i}}=\frac{p_{X_{i}} q_{X_{i}}}{\sigma} ; \pi_{Y_{i}}=\frac{p_{Y_{i}} q_{Y_{i}}}{\sigma}
$$

Market equilibrium leads to

$$
\begin{aligned}
q_{X_{i}} & =\gamma \mu \frac{p_{X_{i}}-\sigma}{K}\left(\frac{I_{i}}{h_{X_{i}} k_{i} p_{X_{i}}^{1-\sigma}+\phi h_{X_{j}} k_{j} p_{X_{j}}^{1-\sigma}}+\phi \frac{I_{j}}{\phi h_{X_{i}} k_{i} p_{X_{i}}^{1-\sigma}+h_{X_{j}} k_{j} p_{X_{j}}^{1-\sigma}}\right) \\
& =\frac{\sigma-1}{\beta_{X_{i}}} \frac{\gamma \mu}{\sigma K}\left(\frac{I_{i}}{h_{X_{i}} k_{i}+\phi h_{X_{j}} k_{j}\left(\frac{\beta_{X_{j}}}{\beta_{X_{i}}}\right)^{1-\sigma}}+\phi \frac{I_{j}}{\phi h_{X_{i}} x_{i}+h_{X_{j}} k_{j}\left(\frac{\beta_{X_{j}}}{\beta_{X_{i}}}\right)^{1-\sigma}}\right) \\
q_{Y_{i}} & =\gamma \mu \frac{p_{Y_{i}}-\sigma}{K}\left(\frac{I_{i}}{h_{Y_{i}} k_{i} p_{Y_{i}}^{1-\sigma}+\phi h_{Y_{j}} k_{j} p_{Y_{j}}^{1-\sigma}}+\phi \frac{I_{j}}{\phi h_{Y_{i}} k_{i} p_{Y_{i}}^{1-\sigma}+h_{Y_{j}} k_{j} p_{Y_{j}}^{1-\sigma}}\right) \\
& =\frac{\sigma-1}{\beta_{Y_{i}}} \frac{I_{i}}{\sigma K}\left(\frac{I_{j}}{h_{Y_{i}} k_{i}+\phi h_{Y_{j}} k_{j}\left(\frac{\beta_{Y_{j}}}{\beta_{Y_{i}}}\right)^{1-\sigma}}+\phi \frac{h_{Y_{i}} x_{i}+h_{Y_{j}} k_{j}\left(\frac{\beta_{Y_{j}}}{\beta_{Y_{i}}}\right)^{1-\sigma}}{\phi h^{-}}\right)
\end{aligned}
$$

so that capital remunerations can be obtained as

$$
\begin{aligned}
& \pi_{X_{i}}=\frac{\gamma \mu}{\sigma K}\left(\frac{I_{i}}{h_{X_{i}} k_{i}+\phi h_{X_{j}} k_{j}\left(\frac{\beta_{X_{j}}}{\beta_{X_{i}}}\right)^{1-\sigma}}+\phi \frac{I_{j}}{\phi h_{X_{i}} k_{i}+h_{X_{j}} k_{j}\left(\frac{\beta_{X_{j}}}{\beta_{X_{i}}}\right)^{1-\sigma}}\right) \\
& \pi_{Y_{i}}=\frac{(1-\gamma) \mu}{\sigma K}\left(\frac{I_{i}}{h_{Y_{i}} k_{i}+\phi h_{Y_{j}} k_{j}\left(\frac{\beta_{Y_{j}}}{\beta_{Y_{i}}}\right)^{1-\sigma}}+\phi \frac{I_{j}}{\phi h_{Y_{i}} k_{i}+h_{Y_{j}} k_{j}\left(\frac{\beta_{Y_{j}}}{\beta_{Y_{i}}}\right)^{1-\sigma}}\right)
\end{aligned}
$$


Taking into account the profit gained in sector $X$ in country $i, \pi_{X_{i}} K_{X_{i}}$, and that in sector $Y$ in country $i, \pi_{Y_{i}} K_{Y_{i}}$, income in country $i$ is given by $I_{i}=\left[\sum_{i=1}^{2}\left(\pi_{X_{i}} K_{X_{i}}+\pi_{Y_{i}} K_{Y_{i}}\right)+L\right]=I_{W} / 2$. The capital remunerations can then be rewritten as

$$
\begin{aligned}
\pi_{X_{1}} & =\frac{\gamma \mu}{\sigma K} \frac{I_{W}}{2}\left[\frac{1}{h_{X_{1}} k_{1}+\phi h_{X_{2}} k_{2} b_{X}}+\phi \frac{1}{\phi h_{X_{1}} k_{1}+h_{X_{2}} k_{2} b_{X}}\right] \\
\pi_{X_{2}} & =\frac{\gamma \mu}{\sigma K} \frac{I_{W}}{2}\left[\phi \frac{1}{h_{X_{1}} k_{1} b_{X}^{-1}+\phi h_{X_{2}} k_{2}}+\frac{1}{\phi h_{X_{1}} k_{1} b_{X}^{-1}+h_{X_{2}} k_{2}}\right] \\
\pi_{Y_{1}} & =\frac{(1-\gamma) \mu}{\sigma K} \frac{I_{W}}{2}\left[\frac{1}{h_{Y_{1}} k_{1}+\phi h_{Y_{2}} k_{2} b_{Y}}+\phi \frac{1}{\phi h_{Y_{1}} k_{1}+h_{Y_{2}} k_{2} b_{Y}}\right] \\
\pi_{Y_{2}} & =\frac{(1-\gamma) \mu}{\sigma K} \frac{I_{W}}{2}\left[\phi \frac{1}{h_{Y_{1}} k_{1} b_{Y}^{-1}+\phi h_{Y_{2}} k_{2}}+\frac{1}{\phi h_{Y_{1}} k_{1} b_{Y}^{-1}+h_{Y_{2}} k_{2}}\right]
\end{aligned}
$$

where

$$
b_{X}=\left(\frac{\beta_{X_{2}}}{\beta_{X_{1}}}\right)^{1-\sigma} ; b_{Y}=\left(\frac{\beta_{Y_{2}}}{\beta_{Y_{1}}}\right)^{1-\sigma}
$$

Given these definitions, country 1 (resp. country 2) has an Absolute Advantage (AA) in the production of good $X$ when $b_{X}<1$ (resp. $\left.b_{X}>1\right)$; country 2 (resp. country 1) has an Absolute Advantage (AA) in the production of good $Y$ when $b_{Y}>1$ (resp. $\left.b_{Y}<1\right)$. By inspection of the above profit expressions, an Absolute Advantage (AA) in a particular sector tends to increase the capital remuneration in that sector. Without loss of generality, we consider country 1 having a Comparative Advantage $(\mathrm{CA})$ in the production of $\operatorname{good} X$, that is, $b_{X}<b_{Y}$. In what follows, we measure the strength of CA by $b \equiv b_{Y} / b_{X}$.

\section{Short run equilibrium}

Given the distribution $k_{i}$ of capital across countries, a short-run equilibrium is defined by some values of $\left(h_{X_{1}}, h_{Y_{1}}, h_{X_{2}}, h_{Y_{2}}\right)$ where $0 \leq h_{X_{i}}, h_{Y_{i}} \leq 1$ and $h_{X_{i}}+h_{Y_{i}}=1$, such that

$$
\begin{aligned}
& \pi_{X_{i}}>\pi_{Y_{i}} \Longrightarrow h_{X_{i}}=1 \\
& \pi_{Y_{i}}>\pi_{X_{i}} \Longrightarrow h_{Y_{i}}=1 \\
& \pi_{Y_{i}}=\pi_{X_{i}} \Longrightarrow 0 \leq h_{X_{i}}, h_{Y_{i}} \leq 1
\end{aligned}
$$

In a short run equilibrium, capital is allocated instantaneously to the sector providing the highest return.

In this paper, we focus on countries having the possibility of fully specializing in some sector. For this purpose, we determine the conditions under which country 1 will specialize in the production of good $X$ and country 2 in the production of good $Y$. When country 1 specializes in $X$ and country 2 in $Y$ 
(that is, $h_{X_{1}}=1, h_{Y_{1}}=0, h_{X_{2}}=0, h_{Y_{2}}=1$ ), capital remunerations reduce to

$$
\begin{aligned}
\pi_{X_{1}} & =\frac{\gamma \mu}{\sigma K} \frac{I_{W}}{2}\left[\frac{1}{k_{1}}+\phi \frac{1}{\phi k_{1}}\right]=\frac{\gamma \mu}{\sigma K} \frac{I_{W}}{2} \frac{2}{k_{1}} \\
\pi_{X_{2}} & =\frac{\gamma \mu}{\sigma K} \frac{I_{W}}{2}\left[\phi \frac{1}{k_{1} b_{X}^{-1}}+\frac{1}{\phi k_{1} b_{X}^{-1}}\right]=\frac{\gamma \mu}{\sigma K} \frac{I_{W}}{2} \frac{1}{k_{1} b_{X}^{-1}} \frac{\phi^{2}+1}{\phi} \\
\pi_{Y_{1}} & =\frac{(1-\gamma) \mu}{\sigma K} \frac{I_{W}}{2}\left[\frac{1}{\phi k_{2} b_{Y}}+\phi \frac{1}{k_{2} b_{Y}}\right]=\frac{(1-\gamma) \mu}{\sigma K} \frac{I_{W}}{2} \frac{1}{k_{2} b_{Y}} \frac{\phi^{2}+1}{\phi} \\
\pi_{Y_{2}} & =\frac{(1-\gamma) \mu}{\sigma K} \frac{I_{W}}{2}\left[\phi \frac{1}{\phi k_{2}}+\frac{1}{k_{2}}\right]=\frac{(1-\gamma) \mu}{\sigma K} \frac{I_{W}}{2} \frac{2}{k_{2}}
\end{aligned}
$$

The full specialization pattern is an equilibrium as long as

$$
\begin{aligned}
& \pi_{X_{1}}>\pi_{Y_{1}}: \frac{\gamma \mu}{\sigma K} \frac{I_{W}}{2} \frac{2}{k_{1}} \geq \frac{(1-\gamma) \mu}{\sigma K} \frac{I_{W}}{2} \frac{1}{k_{2} b_{Y}} \frac{\phi^{2}+1}{\phi} \\
& \pi_{X_{2}}<\pi_{Y_{2}}: \frac{\gamma \mu}{\sigma K} \frac{I_{W}}{2} \frac{1}{k_{1} b_{X}^{-1}} \frac{\phi^{2}+1}{\phi} \leq \frac{(1-\gamma) \mu}{\sigma K} \frac{I_{W}}{2} \frac{2}{k_{2}}
\end{aligned}
$$

that is, if

$$
B_{1}<\frac{k_{2}}{k_{1}}<B_{2}
$$

with

$$
B_{1} \equiv \frac{1}{2} \frac{1-\gamma}{\gamma} \frac{1}{b_{Y}} \frac{\phi^{2}+1}{\phi} ; B_{2} \equiv 2 \frac{1-\gamma}{\gamma} \frac{1}{b_{X}} \frac{\phi}{\phi^{2}+1}
$$

By continuity, country 1 (resp. country 2 ) and only country 1 (resp. country 2 ) will be producing both goods $X$ and $Y$ when $k_{2} / k_{1} \leq B_{1}\left(\right.$ resp. $\left.k_{2} / k_{1} \geq B_{2}\right)$. In order to make full specialization possible, we need $B_{1}<B_{2}$, that is

$$
b \equiv \frac{b_{Y}}{b_{X}}>\left(\frac{\phi^{2}+1}{2 \phi}\right)^{2}
$$

Thus, we need a strong CA of country 1 (resp. country 2) in the production of $X$ (resp. the production of $Y$ ). The condition for full specialization can be rewritten as follows

$$
\frac{1}{2} \frac{1}{b_{Y}} \frac{\phi^{2}+1}{\phi}<\frac{\frac{\gamma \mu I_{W}}{k_{1} K}}{\frac{(1-\gamma) \mu I_{W}}{k_{2} K}}=\frac{\gamma}{1-\gamma} \frac{k_{2}}{k_{1}}<2 \frac{1}{b_{X}} \frac{\phi}{\phi^{2}+1}
$$

Under full specialization, country 1 (resp. country 2) uses all its capital stock $k_{1} K\left(\right.$ resp. $\left.k_{2} K\right)$ in the production of $X($ resp. $Y)$; and the share of expenditures $\gamma \mu I_{W}\left(\right.$ resp. $\left.(1-\gamma) \mu I_{W}\right)$ is spent on good $X($ resp. $\operatorname{good} Y)$. Thus, the ratio of expenditure shares (for a given ratio of capital shares), or the ratio of capital shares (for a given ratio of expenditure shares), has to be within some admissible range. Otherwise, full specialization is simply not possible.

The range of specialization $\left[B_{1}, B_{2}\right]$ is determined by CA and trade costs. 
Proposition 1 The stronger the $C A b$ or the higher the freeness of trade $\phi$, the larger the full specialization range $\left[B_{1}, B_{2}\right]$.

Proof. When there is no trade cost, the range of full specialization is determined by $\mathrm{CA}$ only. The stronger $\mathrm{CA} b \equiv b_{Y} / b_{X}$, the larger the full specialization $\left[B_{1}, B_{2}\right]$, see the similar result by Dornbusch et al (1977). More generally, since $2 \phi /\left(1+\phi^{2}\right)<1$, the condition $B_{1}<B_{2}$ holds when country 1 exhibits a strong $\mathrm{CA}$ in the production of commodity $X$. Moreover, the influence of transport costs can be shown as follows

$$
\begin{aligned}
\frac{\partial B_{1}(\phi)}{\partial \phi} & <0 \text { and } \frac{\partial B_{2}(\phi)}{\partial \phi}>0 \\
B_{1}(\bar{\phi}) & =B_{2}(\bar{\phi}) \text { with } \bar{\phi}=\sqrt{\frac{2-b^{-1}-2 \sqrt{1-b^{-1}}}{b^{-1}}}
\end{aligned}
$$

Therefore, the lower the trade freeness, the smaller the specialization range $\left[B_{1}, B_{2}\right]$ as high trade cost makes local production more attractive. At $\phi=\bar{\phi}$, this range vanishes.

According to Proposition 1, a stronger CA and/or a higher freeness of trade have a positive effect on specialization. This confirms Ricci's (1999) result according to which, for a given market size, an increase in the relative strength of CA - via a direct increase of the competitive advantage or by reducing the importance of local demand - with respect to some agglomeration force favours specialization.

When the capital ratio $k_{2} / k_{1}$ is outside the specialization range, the shares $h_{X_{i}}, h_{Y_{i}}$ can be determined for each $k_{2} / k_{1}$. As $k_{2}=1-k_{1}$, it is convenient to rewrite condition (1) in terms of $k_{1}$ alone. Inserting $k_{2}=1-k_{1}$ shows that full specialisation holds in both countries if

$$
\frac{1}{\frac{2}{b_{X}} \frac{1-\gamma}{\gamma} \frac{\phi}{1+\phi^{2}}+1}<k_{1}<\frac{1}{\frac{1}{2 b_{Y}} \frac{1-\gamma}{\gamma} \frac{1+\phi^{2}}{\phi}+1}
$$

The production shares $h_{X_{i}}, h_{Y_{i}}$ in the two countries are depicted in terms of the capital distribution $k_{1}$ in Figure 1.

As shown in Figure 1, increasing the relative market size represented by the ratio $k_{1} / k_{2}$ could have a 'perverse' effect on specialization with country 1 (resp. 2 ) starting to produce both goods by increasing (resp. decreasing) sufficiently the ratio $k_{1} / k_{2}$. This confirms, in the context of our analysis, Ricci's (1999) result.

As the specialisation pattern clearly depends upon the distribution of capital, we identify the three following ranges:

\begin{tabular}{|l|l|l|}
\hline Range A & $k_{1}<\frac{1}{\frac{2 \phi(1-\gamma)}{\gamma b_{X}\left(\phi^{2}+1\right)}+1}$ & $\begin{array}{l}h_{X_{1}}=1, h_{Y_{1}}=0 \\
0 \leq h_{X_{2}}, h_{Y_{2}} \leq 1\end{array}$ \\
\hline \multirow{2}{*}{ Range B } & $\frac{1}{\frac{2}{b_{X}} \frac{1-\gamma}{\gamma} \frac{\phi}{1+\phi^{2}}+1}<k_{1}<\frac{1}{\frac{1}{2 b_{Y}} \frac{1-\gamma}{\gamma} \frac{1+\phi^{2}}{\phi}+1}$ & $\begin{array}{l}h_{X_{1}}=1, h_{Y_{1}}=0 \\
h_{X_{2}}=0, h_{Y_{2}}=1\end{array}$ \\
\hline \multirow{2}{*}{ Range C } & $k_{1}>\frac{1}{\frac{\left(\phi^{2}+1\right)(1-\gamma)}{2 \phi \gamma b_{Y}}+1}$ & $0 \leq h_{X_{1}}, h_{Y_{1}} \leq 1$ \\
& $h_{X_{2}}=0, h_{Y_{2}}=1$ \\
\hline
\end{tabular}




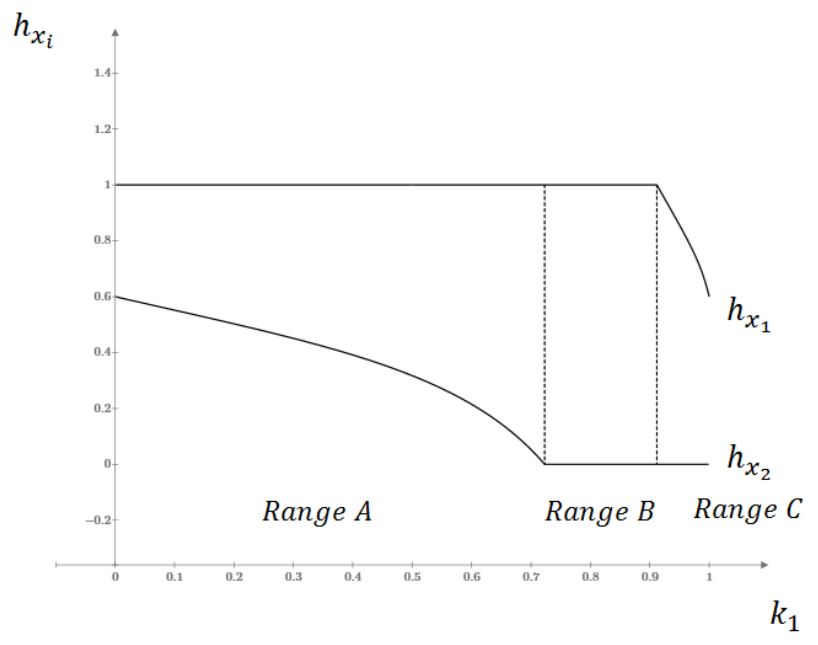

Figure 1: Production shares $h_{x_{1}}$ and $h_{x_{2}}$ in the two countries. The lower (resp. upper) curve corresponds to the production share of commodity $X$ in country 1 (resp. country 2). Parameter values: $\gamma=0.6, \phi=0.4, b_{X}=1.2$ and $b_{Y}=10$.

Range $\mathrm{A}$, where the ratio $k_{1} / k_{2}$ is relatively small, is characterised by country 1 specialising in $X$ and country 2 producing both $X$ and $Y$; range $\mathrm{B}$, where the ratio $k_{1} / k_{2}$ takes intermediate values, by country 1 specialising in $X$ and country 2 specializing in $Y$; finally, range $\mathrm{C}$, where the ratio $k_{1} / k_{2}$ is relatively large, is characterised by country 1 producing both $X$ and $Y$ and country 2 specialising in $Y$.

\section{Dynamics}

In contrast to the instantaneous sectoral relocation assumed in Section 3, we assume that capital adjustments across countries is more costly. These capital movements are driven by a process which resembles the replicator dynamics: capital owners relocate capital from one country to another, if the average profit across sectors differ between countries. Relocation of capital typically involves time delays. A neat way to account for them is a discrete time specification, which we adopt in the following analysis. ${ }^{3}$

\footnotetext{
${ }^{3}$ Note that existence of the fixed points do not depend upon the temporal framework; the stability properties, however, may change.
} 
Expressing the average profit in country $i$ as

$$
\begin{aligned}
\pi_{i, t} & =\left(h_{X_{i}, t} k_{i, t} K \pi_{X_{i}, t}+h_{Y_{i}, t} k_{i, t} K \pi_{Y_{i}, t}\right) /\left(k_{i, t} K\right) \\
& =h_{X_{i}, t} \pi_{X_{i}, t}+h_{Y_{i}, t} \pi_{Y_{i}, t}
\end{aligned}
$$

the adjustment dynamics can be formulated as

$$
\begin{gathered}
\frac{k_{1, t+1}-k_{1, t}}{k_{1, t}}=s\left(1-k_{1, t}\right) \frac{\pi_{1, t}-\pi_{2, t}}{k_{1, t} \pi_{1, t}+\left(1-k_{1, t}\right) \pi_{2, t}} \\
k_{1, t+1}=k_{1, t}\left[1+s\left(1-k_{1, t}\right) \frac{\pi_{1, t}-\pi_{2, t}}{k_{1, t} \pi_{1, t}+\left(1-k_{1, t}\right) \pi_{2, t}}\right]
\end{gathered}
$$

where $s>0$ is the speed of adjustment and $0 \leq k_{1, t+1} \leq 1$.

Since profits depend on the production shares $h_{X_{i}}$ and $h_{Y_{i}}$, the dynamics is defined piecewise on the specialisation ranges. As mentioned earlier, we focus on the range in the parameter space that makes partial and full specialisation possible, that is $b \equiv b_{Y} / b_{X}>\left(1+\phi^{2}\right)^{2} /\left(4 \phi^{2}\right)$.

\subsection{Dynamics in Range $B$}

We start with the dynamics in range $B$, because it is the simplest to derive. Using the profits as given in (5), observing that $h_{X_{1}}=1\left(h_{Y_{1}}=0=h_{X_{2}}\right)$ and $h_{Y_{2}}=1$ and taking into account the constraints on shares, the dynamic equation (6) reduces to

$$
k_{1, t+1}=\left\{\begin{array}{ccc}
0 & \text { if } & Z B<0 \\
Z B & \text { if } & 0 \leq Z B \leq 1 \\
1 & \text { if } & Z B>1
\end{array}\right.
$$

where

$$
Z B=(1-s) k_{1, t}+\gamma s
$$

is a linear difference equation or, in the language of dynamical systems theory, a linear map.

Proposition 2 If $b_{X}<\frac{2 \phi}{\phi^{2}+1}<1<\frac{\phi^{2}+1}{2 \phi}<b_{Y}$, there is a unique fixed point $k_{1}^{f i x B}=\gamma$ in range $B$. The fixed point is stable if $-1<1-s<1$, that is, if $s<2$. At $s=2$ the fixed point loses stability via the threshold -1 .

Proposition 2 says that the fixed point in range $B$ exists if country 1 has a strong Absolute Advantage (AA) in the production of $X$ and country 2 a strong AA in $Y$. Also, a lower speed of adjustment $s$ favors the stability of this fixed point. 


\subsection{Dynamics in Range A}

The dynamics in the other regions is more complicated, but can still be stated explicitly. In range $\mathrm{A}$, in which $h_{X_{1}}=1, h_{Y_{1}}=0$ and $0 \leq h_{X_{2}}, h_{Y_{2}} \leq 1$ holds, the dynamics is as follows:

$$
k_{1, t+1}=\left\{\begin{array}{ccc}
0 & \text { if } & Z A<0 \\
Z A & \text { if } & 0 \leq Z A \leq 1 \\
1 & \text { if } & Z A>1
\end{array}\right.
$$

where

$$
Z A=k_{1, t}\left[1+s\left(1-k_{1, t}\right)\left(G-\frac{1-\gamma}{1-k_{1, t}}\right)\right]
$$

is a nonlinear (unimodal) difference equation or map; and where

$$
G=\frac{\gamma}{2} \frac{-2 \phi b_{X}^{2}\left(1-k_{1, t}\right) h_{X_{2}, t}^{2}+b_{X}\left(\phi^{2}+1\right)\left(1-2 k_{1, t}\right) h_{X_{2}, t}+2 \phi k_{1, t}}{\phi k_{1, t}^{2}+b_{X} k_{1, t}\left(\phi^{2}+1\right)\left(1-k_{1, t}\right) h_{X_{2}, t}+\phi b_{X}^{2}\left(1-k_{1, t}\right)^{2} h_{X_{2}, t}^{2}}
$$

The production share $h_{X_{2}, t}$ can be explicitly determined from the short-run equilibrium condition $\pi_{X_{2}, t}=\pi_{Y_{2}, t}$, which solves for two roots; it can be shown analytically that only one is in the admissible range $[0,1]$, that is:

$$
h_{X_{2}, t}=\left(\frac{\gamma}{2}+\frac{k_{1, t}\left(\phi^{2}+1\right)(\gamma-2)+\sqrt{R+S}}{4 \phi\left(1-k_{1, t}\right) b_{X}}\right)
$$

with

$$
\begin{gathered}
R=4 \gamma^{2} \phi\left(1-k_{1, t}\right)\left(\phi\left(1-k_{1, t}\right) b_{X}^{2}+k_{1, t}\left(\phi^{2}+1\right) b_{X}\right) \\
S=k_{1, t}^{2}\left(\phi^{2}+1\right)^{2}\left(\gamma^{2}+4(\phi-1)^{2}(1-\gamma)\right)
\end{gathered}
$$

\section{Proposition 3 If}

$$
\frac{2 \phi}{\phi^{2}+1}<b_{X}<\frac{\phi^{2}+1}{2 \phi}
$$

there exists an interior fixed point in range $A$ given by

$$
\begin{gathered}
k_{1}^{f i x A}=\frac{\gamma b_{X}\left(\frac{\phi^{2}+1}{2 \phi}-b_{X}\right)}{\left(\phi-b_{X}\right)\left(b_{X}-\frac{1}{\phi}\right)} \\
h_{X_{2}}^{f i x A}=\frac{\gamma\left(\phi^{2}+1\right)\left(\frac{2 \phi}{\phi^{2}+1}-b_{X}\right)}{2 \phi(1-\gamma)\left(b_{X}\left(b_{X}-\frac{2-\gamma}{1-\gamma} \frac{\phi^{2}+1}{2 \phi}\right)+\frac{1}{1-\gamma}\right)} \\
\text { with } \quad \frac{\partial k_{1}^{f i x A}}{\partial b_{X}}<0 \quad \frac{\partial k_{1}^{f i x A}}{\partial \phi}<0 \\
\frac{\partial k_{1}^{f i x A}}{\partial \phi}>0 \quad \text { if } \quad b_{X}>1
\end{gathered}
$$


Proof. Note that in a fixed point short-run and long-run equilibrium conditions have to be satisfied simultaneously. Therefore, the fixed point is defined by $\pi_{X_{1, t}}=\pi_{X_{2}, t}=\pi_{Y_{2}, t}$, which can be solved for $k_{1}^{f i x A}$ and $h_{X_{2}}^{f i x A}$. Exploiting $0<k_{1}^{f i x A}<\frac{1}{\frac{2 \phi(1-\gamma)}{\gamma b X\left(\phi^{2}+1\right)}+1}$ and $0<h_{X_{2}}^{\text {fixA }}<1$ results in the parameters range given in the proposition.

Note that condition (7) is satisfied, if either country has a weak AA in good $X$ as $b_{X}$ can be less or greater than 1 . However, combining condition (7) and condition (2) shows that the interior fixed point in range A requires that country 2 has an AA in good $Y$.

Corollary 4 It is easily checked that at the fixed point $A$ the following relation holds:

$$
\left(1-k_{1}^{f i x A}\right)\left(1-h_{X_{2}}^{f i x A}\right)=k_{2}^{f i x A} h_{Y_{2}}^{f i x A}=1-\gamma
$$

No analytical stability properties are available. However, in the simulations, the map $Z A$ proves to be isomorphic to the well-known logistic map: for low adjustment speeds it exhibits a positive slope (but less than +1 ) at the fixed point; increasing the adjustment speed reduces the slope at the fixed point until the fixed point loses stability via a Flip-bifurcation (when the slope crosses the -1 threshold).

Proposition 5 In addition, there is a Core-Periphery fixed point in range $A$ given by $k_{1}^{C P A}=0$. It is locally stable if $b_{X}>\frac{\phi^{2}+1}{2 \phi}(>1)$; i.e. if the $A A$ country 2 has in $X$ is sufficiently strong.

\subsection{Dynamics in Range $\mathrm{C}$}

In Region $C$, we have $h_{X_{2}}=0, h_{Y_{2}}=1$ and $0 \leq h_{X_{1}}, h_{Y_{1}} \leq 1$. The dynamics that governs capital relocation is determined by the following equation:

$$
k_{1, t+1}=\left\{\begin{array}{ccc}
0 & \text { if } & Z C<0 \\
Z C & \text { if } & 0 \leq Z C \leq 1 \\
1 & \text { if } & Z C>1
\end{array}\right.
$$

where

$$
Z C=k_{1, t}\left[1+s \frac{1-k_{1, t}}{2 k_{1, t}} \frac{2 W-X(1+\gamma)+2 \gamma \phi b_{Y}^{2}}{W-X+\phi b_{Y}^{2}}\right]
$$

is a nonlinear map; and where

$$
\begin{aligned}
W & =k_{1, t}^{2}\left(b_{Y}+\phi\left(h_{X_{1}, t}-1\right)\right)\left(\phi b_{Y}+h_{X_{1}, t}-1\right) \\
X & =b_{Y} k_{1, t}\left(\left(\phi^{2}+1\right)\left(h_{X_{1}, t}-1\right)+2 \phi b_{Y}\right)
\end{aligned}
$$

The production share $h_{X_{1}, t}$ can be explicitly determined from the short-run equilibrium condition $\pi_{X_{1}, t}=\pi_{Y_{1}, t}$, which solves for two roots; it can be shown 
analytically that the only one in the admissible range $[0,1]$ is:

$$
h_{X_{1}, t}=\left(\frac{1+\gamma}{2}+\frac{(1+\gamma)\left(1-k_{1, t}\right)\left(\phi^{2}+1\right) b_{Y}-\sqrt{U+V}}{4 \phi k_{1, t}}\right)
$$

with

$$
\begin{gathered}
U=b_{Y}^{2}\left(1-k_{1, t}\right)^{2}\left[(1-\gamma)^{2}\left(\phi^{2}+1\right)^{2}+4 \gamma\left(\phi^{2}-1\right)^{2}\right] \\
V=4 \phi k_{1, t}(1-\gamma)^{2}\left[\left(\phi^{2}+1\right)\left(1-k_{1, t}\right) b_{Y}+\phi k_{1, t}\right]
\end{gathered}
$$

Proposition 6 If

$$
\frac{2 \phi}{\phi^{2}+1}<b_{Y}<\frac{\phi^{2}+1}{2 \phi}
$$

there exists an interior fixed point in range $C$ given by

$$
\begin{aligned}
k_{1}^{f i x C} & =\frac{\left(\phi^{2}+1\right)(\gamma+1)\left(b_{Y}-\frac{\gamma}{\gamma+1} \frac{2 \phi}{\phi^{2}+1}\right)-2 \phi b_{Y}^{2}}{2 \phi\left(\phi-b_{Y}\right)\left(b_{Y}-\frac{1}{\phi}\right)} \\
h_{X_{1}}^{f i x C} & =\frac{\gamma}{k_{1}^{f i x C}} \\
\frac{\partial k_{1}^{f i x C}}{\partial b_{Y}}<0 & \\
\frac{\partial k_{1}^{f i x C}}{\partial \phi}>0 & \text { if } \quad b_{Y}>1 \\
\frac{\partial k_{1}^{f i x C}}{\partial \phi}<0 & \text { if } \quad b_{Y}<1
\end{aligned}
$$$$
\text { with } \quad \frac{\partial k_{1}^{f i x C}}{\partial \phi}>0 \quad \text { if } \quad b_{Y}>1
$$

Proof. Note that in a fixed point, both short-run and long-run equilibrium conditions have to hold simultaneously. Therefore, the fixed point is defined by $\pi_{Y_{1, t}}=\pi_{Y_{2}, t}=\pi_{X_{1}, t}$, which can be solved for $k_{1}^{\text {fixC }}$ and $h_{X_{2}}^{\text {fix } C}$. Exploiting $\frac{1}{\frac{\left(\phi^{2}+1\right)(1-\gamma)}{2 \phi \gamma b_{Y}}+1}<k_{1}^{f i x C}<1$ and $0<h_{X_{1}}^{f i x C}<1$, we obtain the results stated in the proposition.

No result with respect to stabiltiy properties is available. However, in simulations, we found instances of locally stable fixed points.

Proposition 7 A Core-Periphery fixed point exists in range $C$ with $k_{1}^{C P C}=1$. It is locally stable if $b_{Y}<\frac{2 \phi}{\phi^{2}+1}(<1)$

Given that the dynamics in range $C$ is pretty much symmetric to that in range $A$, the following analysis focusses on the dynamics in regions $A$ and $B$.

\subsection{Effects of Comparative and Absolutes Advantages on Long-Run Equilibria}

As stressed above, we focus our analysis on the case where at least one country fully specialises, i.e. where country 1 (resp. country 2) exhibits a strong CA in 
the production of commodity $X$ (resp. $Y)$, that is, $\frac{b_{Y}}{b_{X}}>\left(\frac{\phi^{2}+1}{2 \phi}\right)^{2}$. First, we look at the role of $\mathrm{CA}$ and $\mathrm{AA}$ on the long-run distribution of capital. For this purpose, we analyze the long-run dynamics for increasing values of $b_{X}$. As $b_{X}$ increases, country 1 loses progressively the AA it has in good $X$ at the expense of country 2. Note that as strong CA is assumed even for high values of $b_{X}$, this implies that country 2 has a strong AA in good $Y$; for $b_{X}>1$ it has also an AA in good $X$. Results, that have been derived analytically, are illustrated in Figure 2.

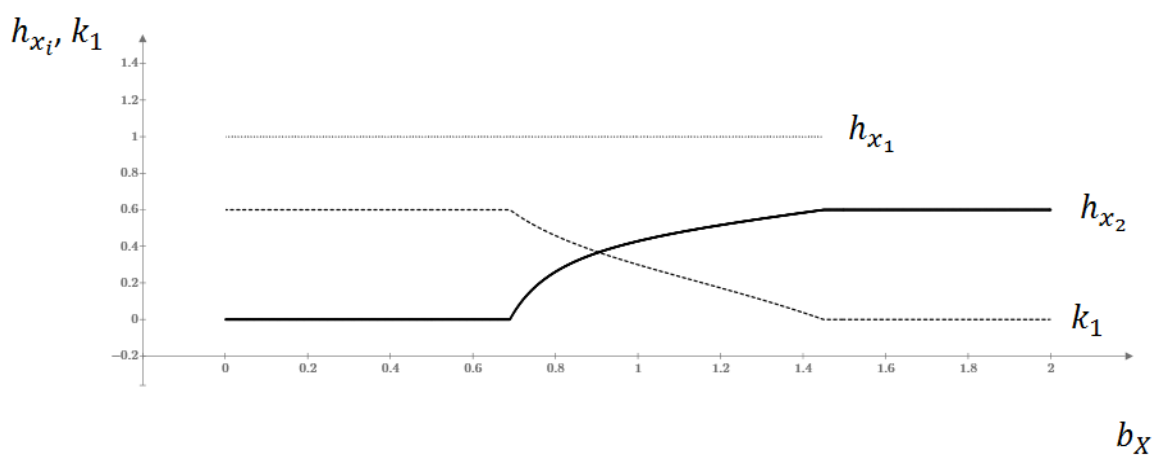

Figure 2: The dashed curve represents the share of capital $k_{1}$ in region 1 and the dotted (resp. solid) curve the production share $h_{X_{1}}\left(\right.$ resp. $\left.h_{X_{2}}\right)$ of commodity $X$ in region 1 (resp. region 2). Parameter values: $\gamma=0.6, \phi=0.4$ and $b_{Y}=10$.

For low values of $b_{X}<\frac{2 \phi}{\phi^{2}+1}<1<\frac{\phi^{2}+1}{2 \phi}<b_{Y}$ indicating a strong AA of country $1(2)$ in good $X(Y)$, the long-run equilibrium belongs to regime $B$ as stated by Proposition 2: each country holds capital and specializes according to its CA. For high values of $b_{X}>\frac{\phi^{2}+1}{2 \phi}(>1)$ indicating an AA of country 2 in good $X$ as well as in good $Y$, the long-run equilibrium belongs to regime $A$ as stated by Propositions 4: economic activity ends up concentrating in country 2. In this case, both goods are produced in country 2 and country 1 has lost all its manufacturing capital $\left(k_{1}=0\right.$ and thus no $h_{X_{1}}$ is observable - i.e. the dotted line in Figure 2 vanishes). For intermediate values of $b_{X}\left(\frac{2 \phi}{\phi^{2}+1}<b_{X}<\frac{\phi^{2}+1}{2 \phi}\right.$ corresponding to either country 1 or 2 having a weak AA in good $X$ ), country 1 specializes in good $X$ according to its $\mathrm{CA}$ while country 2 produces both goods. Increasing $b_{X}$, more capital is moving to country 2 , and more varieties of good $X$ are produced in country 2 so as to compensate for the decrease in varieties in country 1 . So, when a country has a strong $A A$ in both goods, it attracts the entire manufacturing activity. When each country has an $A A$ in the good in which it has a $C A$, specialisation is driven by $C A$.

Next, we turn to a standard exercise in the NEG literature, we investigate the effect of trade freeness on long run equilibria. We show that it crucially 
depends on the intensity of AA. We continue to focus our analysis on the case where country 1 (resp. country 2) exhibits a strong CA in the production of commodity $X$ (resp. $Y)$, that is, $\frac{b_{Y}}{b_{X}}>\left(\frac{\phi^{2}+1}{2 \phi}\right)^{2}$. Note that this condition can be transformed into $\phi>\sqrt{b_{Y} / b_{X}}-\sqrt{b_{Y} / b_{X}-1}$, which sets a lower bound for the trade freeness. Thus, our analysis assumes a relatively high trade freeness. Note in addition, that the conditions for the existence of $k_{1}^{f i x A}$ set an upper bound for the trade freeness which are the following:

$$
\begin{array}{ccc}
\phi<b_{X}-\sqrt{b_{X}^{2}-1} \quad \text { if } \quad b_{X}>1 & \frac{\partial k_{1}^{f i x A}}{\partial \phi}<0 \\
\phi<\frac{1-\sqrt{1-b_{X}^{2}}}{b_{X}} \quad \text { if } \quad b_{X}<1 & \frac{\partial k_{1}^{f i x A}}{\partial \phi}>0
\end{array}
$$

Two typical scenarios emerge :

- In the case of $b_{X}<1$, i.e. in the case in which country 1 has an AA in the production of $X$, and starting with $\phi$ at its lower boundary $\sqrt{b_{Y} / b_{X}}-\sqrt{b_{Y} / b_{X}-1}$, we find a fixed point in region $A$; country 1 specialises in the production of $X$ and country 2 produces both manufatured commodities. Increasing the trade freeness $\phi$, increases the $k_{1}^{f i x A}$, country 1 gains capital and beyond $\phi=\frac{1-\sqrt{1-b_{X}^{2}}}{b_{X}}$ the fixed point enters region $B$ and both countries specialise in one commodity: Country 1 continues to specialises in $X$, whereas country 2 has reduced the share of $X$ and continously increased the share of $Y$; and beyond the threshold it produces only $Y$ ). These analytic findings are summarized in Figure 3.

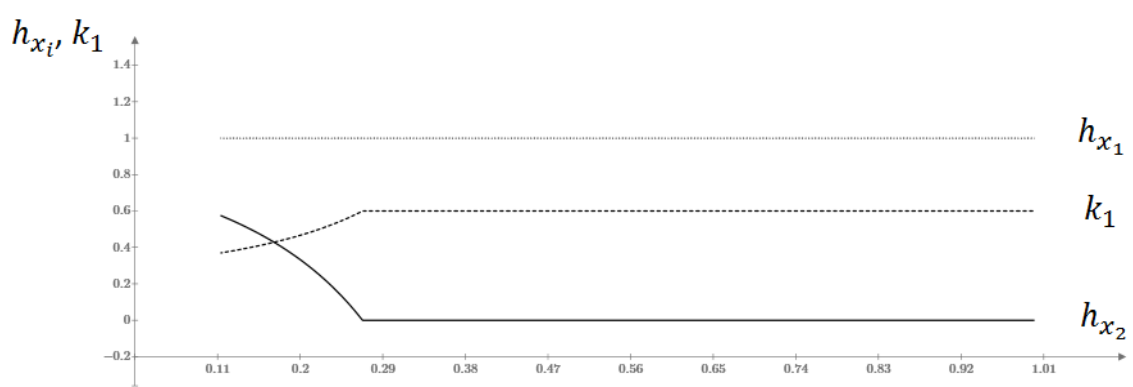

Figure 3: Long-run equilibrium for $b_{X}<1$ in terms of $\phi$. The dashed curve represents the share of capital in region 1 ; the dotted (resp. solid) curve represents the production share $h_{X_{1}}$ (resp. $h_{X 2}$ ) of commodity $X$ in region 1 (resp. region 2). Parameter values: $\gamma=0.6, b_{X}=0.5$ and $b_{Y}=10$.

When country 1 has an AA in $X$ and country 2 in $Y$, the higher the freeness of trade, the higher the capital share of country 1. See Figure 3 where regime 
$A$ holds for low $\phi<\frac{1-\sqrt{1-b_{X}^{2}}}{b_{X}}$ and regime $B$ for high $\phi$. When countries have both a $C A$ and an $A A$ in some good, very low trade costs induce a dispersion of capital across countries and specialization is driven by $C A$ as the benefit from $C A$ dominates market size effects. The result of Forslid and Wooton (2003) is thus confirmed.

- In the case of $b_{X}>1$, i.e. in the case in which country 2 has an AA in the production of $X$ as well as in the production of $Y$, starting with $\phi$ at its lower boundary $\sqrt{b_{Y} / b_{X}}-\sqrt{b_{Y} / b_{X}-1}$, we find a fixed point in region $A$; country 1 specialises in the production of $X$, while country 2 produces both commodities. Increasing the trade freeness, $k_{1}^{\text {fix } A}$ shrinks until finally - at $\phi=b_{X}-\sqrt{b_{X}^{2}-1}$ - country 1 has lost all its industry and industrial production is agglomerated in country 2 that continues to produce both commodities. Figure 4 summarizes these findings (note that for $\phi>b_{X}-\sqrt{b_{X}^{2}-1}$ no industrial production occurs in country 1 and thus the dotted line vanishes in Figure 4).

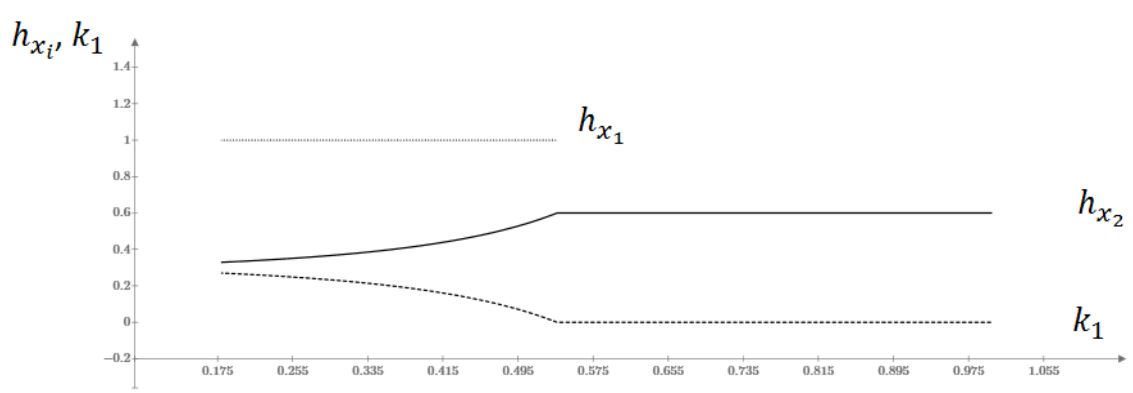

Figure 4: Long run equilibrium for $b_{X}>1$ in terms of $\phi$. The dashed curve represents the share of capital in region 1 ; the dotted (resp. solid) curve represents the production share $h_{X_{1}}\left(\right.$ resp. $h_{X_{2}}$ ) of commodity $X$ in region 1 (resp. region 2). Parameter values: $\gamma=0.6, b_{X}=1.2$ and $b_{Y}=10$.

When country 2 has an AA in both $X$ and $Y$, the higher the freeness of trade $\phi$, the more concentrated the economic activity in country 2. See Figure 4 where regime $A$ holds for all values of $\phi$. Lower trade costs, i.e. $\phi>b_{X}-$ $\sqrt{b_{X}^{2}-1}$, make the location of production less relevant and the benefit from $\mathrm{CA}$ smaller so that capital and economic activity agglomerates in the most productive country. So, when trade costs are very low, a country having an AA in both goods attracts all capital as the market size effect dominates the benefit from $C A$. In contrast to symmetric core-periphery models, here AA determines the location of agglomeration. The fact that country 2 enjoys AA in both sectors explains why the result of Forslid and Wooton (2003), according to which high trade freeness leads to dispersion of the economic activity, is not confirmed. 


\subsection{Results for higher order fixed points and bifurcations with respect to the speed of adjustment}

So far, our analysis has concentrated on locally stable equilibria: assuming a sufficiently small speed of adjustment $s$ - depending also on the initial condition -, the system converges to an interior equilibrium with dispersion of economic activity or to a Core-Periphery equilibrium with capital fully agglomerated in one country. The adjustment towards a long-run equilibrium is progressive or might involve slight overshooting. However, by increasing the speed of adjustment $s$, overshooting intensifies and given the piecewise definition of the map - including the flat branches (resulting from the boundaries at zero and one) and its strong nonlinearity in ranges $A$ and $C$-, it is not surprising that in our discrete-time set up, the dynamics of the main state variable - the capital share $k_{1, t}$ - easily exhibits persistent regular or irregular trajectories. In particular, in simulations (not presented in the text), by varying the adjustment speed $s$, we have detected the following phenomena:

- Given that the map $Z A$ is isomorphic to the logistic map, the fixed point / stationary equilibrium loses stability via a so-called period doubling bifurcation sequence (i.e. the emergence of stable cycles whose periodicity doubles as the speed of adjustment $s$ is increased until an accumulation point is reached) giving rise to the typical cascade towards chaotic attractors. It is worth noticing that the attractor (i.e. a regular or an irregular cycle) may cross the boundaries of range $A$ with a qualitative change along its long-run trajectory.

- The map in range $B$ is linear; however, due to the overall piecewise definition of the map, cyclical or chaotic attractors appear after the fixed point has lost stability. Note that these attractors necessarily cross the boundaries of range $\mathrm{B}$ entering in one or both of the other two ranges changing their qualitative behaviour.

- Due to the boundary conditions (zero and one on the capital share $k_{1, t}$ ), the map may involve one or two flat branches (mapping into CP equilibria). This implies that locally unstable CP equilibria may still enjoy global stability. In other words, both CP equilibria may have a non empty basin of attraction even if they are locally unstable (which could occur via a so-called border collision bifurcation).

- We also find instances of multistability, i.e. coexistence of cyclical and/or chaotic attractors.

Therefore, the map of our model is able to generate very rich and diverse dynamics. However, given the constraints on the paper lenght, we leave a proper investigation for further research. 


\section{Conclusion}

This paper is motivated by the following stylized facts: as developing countries engage further in trade, they tend to specialize in specific ranges of manufactured goods; industrial production and its location are affected by firms' market power and decreasing average costs, especially as productive factors become increasingly mobile between countries.

We constructed a model to study the role of classical trade and NEG elements on the long run spatial distribution of economic activity. For that purpose, we used a Footloose Capital model incorporating labour productivities varying across sectors and countries so that countries enjoy comparative and absolute advantages. With the evidence on multi cones of specialisation in mind, our analysis focused on the parameter range for which at least a country fully specialises in a single industrial sector. We showed that in the short run, that is, for a given spatial distribution of productive factors, specialisation patterns are shaped by comparative advantage. In the long run, when factor mobility is accounted for, absolute advantage was shown to be crucial in determining the location of economic activity. If each country has an absolute advantage in some industrial sector, then further integration (as corresponding to a reduction in trade costs) favours dispersion of industrial capital across countries, and each country specialises according to comparative advantage. If, instead, a country benefits from a superior technology and thus from an absolute advantage in both sectors, then further integration leads to a core periphery structure, with manufacturing production leaving the country with the lagging technology. Interestingly enough, absolute advantage and its interaction with market size effects shape the long run economic geography of a world with multi cones of specialisation.

Acknowledgment: We would like to thank an anonymous referee whose comments have helped us to improve our work and its presentation. We would also like to mention that this work has been performed under the auspices of COST Action IS1104 "The EU in the new complex geography of economic systems: models, tools and policy evaluation".

\section{References}

Bagoulla, C. (2006). Localisation industrielle et spécialisation. Les rôles des infrastructures, des coûts de production et de la taille de marché dans un cadre Nord Sud. Revue économique, 57(4), 705-726.

Baldwin, R., Forslid, R., Martin, Ph., Ottaviano, O. \& F. Robert-Nicoud (2005). Economic Geography and Public Policy, Princeton University Press.

Dornbusch, R., S., Fischer, S, \& Samuelson, P.A. (1977). Comparative Advantage, Trade, and Payments in a Ricardian Model with a Continuum of Goods. American Economic Review, 67, 823-839. 
Fujita M, Krugman P., \& Venables A J, (1999). The Spatial Economy: Cites, Regions and International Trade (MIT Press, Cambridge, MA).

Huang, Y., \& Lee, C., \& Huang, D. (2014). Home Market Effects in the Chamberlinian-Ricardian World. Bulletin of Economic Research, 66(S1), S36S54.

Forslid, R., \& Wooton, I. (2003). Comparative Advantage and the Location of Production. Review of International Economics, 11(4), 588-603.

Head, K. \& T. Mayer (2004). The Empirics of Agglomeration and Trade, chapter 59 in the Handbook of Regional and Urban Economics, vol. 4, edited by V. Henderson and J.-F. Thisse, Elsevier.

Krugman, P. (1991). Increasing Returns and Economic Geography. The Journal of Political Economy, 99(3), 483-499.

Krugman, P. (2008). Trade and Wages, Reconsidered. Brookings Papers on Economic Activity, Spring 2008, 103-137.

Kiyota, k. (2014). A many-cone world? Journal of International Economics, 86, 345-354.

Leamer, Edward E., (1987). Paths of development in the three-factor, ngood general equilibrium model. Journal of Political Economy, 95 (5), 961-999.

Martin, P., \& Rogers, C. A. (1995). Industrial location and public infrastructure. Journal of international Economics, 39(3), 335-351.

Matsuoka, Y., \& Kikuchi, T. (2012). Footloose Capital and Comparative Advantage. Pacific Economic Review, 17(5), 677-686.

Pflüger, M., \& Tabuchi, T. (2016). Comparative Advantage and Agglomeration of Economic Activity. IZA Discussion Paper No. 10273.

Ricci, L. A. (1999). Economic geography and comparative advantage: Agglomeration versus specialization. European Economic Review, 43, 357-377. 